\title{
Subcellular Localization of a Germiantion-specific Cortex-lytic Enzyme, SleB, of Bacilli during Sporulation
}

\author{
Atsushi Masayama ${ }^{1}$, Hideyuki Fukuoka ${ }^{1}$, Shiro Kato ${ }^{1}$, Tohru Yoshimura ${ }^{1}$, \\ Michie Moriyama ${ }^{2}$ and Ryuichi Moriyama ${ }^{3 *}$ \\ ${ }^{1}$ Department of Applied Molecular Biosciences, Graduate School of Bioagricultural Sciences, \\ Nagoya University, Chikusa, Nagoya, Aichi 464-8601, Japan \\ ${ }^{2}$ Department of Home Economics, Aichi Gakusen University, 28 Kamikawanari, \\ Hegoshi-cho, Okazaki, Aichi 444-8520, Japan \\ ${ }^{3}$ Department of Food and Nutritional Sciences, College of Bioscience and \\ Biotechnology, Chubu University, 1200 Matsumoto-cho, \\ Kasugai, Aichi 487-8501, Japan
}

(Received 24 April 2006, accepted 2 June 2006)

\begin{abstract}
The subcellular localization of a germination-specific cortex-lytic enzyme, SleB, of Bacillus subtilis during sporulation was observed by using fusions of N-terminal region of SleB to the green fluorescent protein (GFP). A fusion with a putative peptidoglycan-binding motif $\left(\mathrm{SleB}_{1-108}\right.$-GFP) formed a fluorescent ring around the forespore of the wild type strain, as expected from the known location of the intact $\mathrm{SleB}$ in the dormant spore. $\mathrm{SleB}_{1-108}$-GFP formed a similar fluorescent ring around the forespore of the gerE mutant which has a severe defect in the coat structure, and of the $c w l D$ mutant which lacks a muramic $\delta$-lactam unique to the spore peptidoglycan (cortex), whereas the fusion could not attach to the spore of the cwlDgerE mutant. By contrast, a fusion without the motif (SleB ${ }_{1-45}-\mathrm{GFP}$ ) could not be recruited around the forespore of the gerE mutant though it appeared to be accumulated on the outside of the spore of the wild type strain. Since SleB was shown to degrade only the cortex with muramic $\delta$-lactam, these results suggested that a proper localization of SleB requires a strict interaction between the motif of the enzyme and the $\delta$-lactam structure of the cortex, not the formation of normal coat layer.
\end{abstract}

Key words: bacterial spore, subcellular localization, peptidoglycan-binding motif, germination enzyme, Bacillus subtilis

\section{INTRODUCTION}

Bacterial spore germination, defined as the irreversible loss of spore dormancy, is triggered by specific germinants and proceeds through a set of sequential steps. Spore germination is essential to allow spore outgrowth and the formation of a new vegetative cell; once triggered, this process proceeds in the absence of germinants and germinant-stimulated metabolism. This fact indicates that spore germination is a process controlled by the sequential activation of a set of preexisting germinationrelated enzymes, but not by protein synthesis (Foster and Johnstone, 1990, Moir and Smith, 1990).

One of the key enzymes involved in germination pro-

Edited by Fujio Kawamura

* Corresponding author. E-mail: moriyama@isc.chubu.ac.jp cess is a lytic enzyme specific to an intact spore cortex. Such enzymes had been identified in the spores of Bacillus megaterium KM (GSLE) (Foster and Johnstone, 1987), Bacillus cereus IFO13597 (SleB) (Makino et al., 1994, Moriyama et al., 1996b), Bacillus subtilis 168 (SleB) (Moriyama et al., 1996a) and Clostridium perfringens S40 (SleC) (Miyata et al., 1995a, Miyata et al., 1995b). B. cereus $\mathrm{SleB}$ degrades only spore cortex peptidoglycan that contains muramic $\delta$-lactam unique to the cortex, but not the peptidoglycan of vegetative cell walls (Makino et al., 1994, Miyata et al., 1995b). B. subtilis cwlD spores which completely lack muramic $\delta$-lactam in their cortex are unable to complete germination through the blocking of cortex degradation (Sekiguchi et al., 1995, Popham et al., 1996). These results suggest that muramic $\delta$-lactam could be a substrate recognition determinant for SleB (Atrih et al., 1998, Makino and Moriyama, 2002). B. 
subtilis and B. cereus $\mathrm{SleB}$ and $C$. perfringens $\mathrm{SleC}$ contain the characteristic motif consisting of tandem repeat sequences at its $\mathrm{N}$-terminal domain, which is conserved in noncatalytic regions of various peptidoglycan hydrolases with a diversity of substrate specificities (Moriyama et al., 1996b). The motif was suggested to be involved in the recognition of some repeated unit of peptidoglycan of the cell wall and spore cortex (Moriyama et al., 1996b).

The sleB gene, which was shown to be crucial in germination of $B$. subtilis spores (Moriyama et al., 1996a, Boland et al., 2000), is conserved among other Bacillus species, such as B. cereus, $B$. anthracis, $B$. halodurans, $B$. megaterium, and Oceanobacillus iheyensis (Makino and Moriyama, 2002). B. subtilis sleB is expressed as an operon with a 3 '-adjacent ypeB gene in the forespore compartment of sporangia under the control of $\sigma^{\mathrm{G}}$, a sporulation-specific sigma factor (Moriyama et al., 1999). Immunoelectron microscopic localization of $B$. subtilis and $B$. cereus SleB proteins just inside the spore coat layer in the dormant spores suggested that the enzymes are translocated across the inner forespore membrane by a secretion signal peptide, and deposited in spore cortex synthesized between the inner and outer forespore membranes during sporulation (Moriyama et al., 1999). However, further investigations should be conducted to obtain an understanding of the molecular mechanism of the subcellular localization of SleB in order to reveal the process by which the molecular construction of germination apparatus during sporulation takes place.

In the present study, we observed the localization patterns of B. subtilis SleB during sporulation by using fusions of N-terminal region of SleB to the green fluorescent protein (GFP), and examined the effects of sporulation defects and a deletion of the tandem repeat sequences of the enzyme on the localization of the fusion. The results suggested that SleB requires the interaction between the motif of the enzyme and muramic $\delta$-lactam of the spore cortex for its proper localization.

\section{MATERIALS AND METHODS}

Bacterial strains and growth condition The bacterial strains used in this study are listed in Table 1. Escherichia coli and B. subtilis strains were grown on Luria-Bertani broth or agar at $37^{\circ} \mathrm{C}$. B. subtilis was transformed as previously described (Moriyama et al., 1999). If necessary, antibiotics were added in the following concentrations: ampicillin $\left(100 \mu \mathrm{g} \mathrm{ml}^{-1}\right)$, chloramphenicol $\left(5 \mu \mathrm{g} \mathrm{ml}^{-1}\right)$, tetracycline $\left(20 \mu \mathrm{g} \mathrm{ml}^{-1}\right)$.

Spore preparation $B$. subtilis strains were sporulated at $30^{\circ} \mathrm{C}$ or $37^{\circ} \mathrm{C}$ by nutrient exhaustion in Difco sporulation medium (Schaeffer et al., 1965). The spores were harvested and purified by being washed with cold distilled water until the cell debris and vegetative cells had
Table 1. B. subtilis strains and plasmids

\begin{tabular}{|c|c|c|}
\hline Strain or plasmid & Genotype or description & Source or reference \\
\hline \multicolumn{3}{|l|}{ B. subtilis strains } \\
\hline PY79 & Wild type & A. Driks \\
\hline $\mathrm{AD} 17$ & gerE36 & A. Driks \\
\hline ADD1 & purB his-1 smo-1 cwlD::cat & J. Sekiguchi \\
\hline HF17 & gerE36 cwlD::cat & This study \\
\hline HF18 & gerE36 cwlD::cat pHYS108G & pHYS108G $\rightarrow$ HF17 \\
\hline RM1 & pHYS108G & pHYS108G $\rightarrow$ PY79 \\
\hline RM2 & pHYS45G & pHYS45G $\rightarrow$ PY79 \\
\hline RM3 & gerE36 pHYS108G & pHYS108G $\rightarrow$ AD17 \\
\hline RM4 & gerE36 pHYS45G & $\mathrm{pHYS} 45 \mathrm{G} \rightarrow \mathrm{AD} 17$ \\
\hline RM5 & $\begin{array}{l}\text { purB his-1 smo-1 cwlD::cat } \\
\text { pHYS108G }\end{array}$ & pHYS108G $\rightarrow$ ADD1 \\
\hline \multicolumn{3}{|l|}{ Plasmids } \\
\hline pBluescriptII SK(-) & lacZ bla & Stratagene \\
\hline pBT374 & lacZ bla cwlD-cat & J. Sekiguchi \\
\hline pHY300PLK & bla tet & Takara \\
\hline pHYG1 & bla tet $g f p$ & 13 \\
\hline pHYS108G & bla tet $s l e B_{1-108-g f p}$ & This study \\
\hline pHYS45G & bla tet sleB $B_{1-45^{-}}$gfp & This study \\
\hline
\end{tabular}

been removed.

Construction of sleB-gfp fusions Plasmid pHYS108G and pHYS45G were constructed as follows. B. subtilis sleB was obtained by PCR amplification from pBS45H as a DNA segment containing the sleB promoter region and Met-1 to Val-108 or Met-1 to Val-45 of SleB, using as primers oligonucleotides that created a HindIII site at the 5' end and a SalI site at the 3' end. The PCR fragments were digested with HindIII and SalI and then ligated to pBluescriptII SK(-) (Stratagene) that had been digested with HindIII and SalI, yielding pBSL108 and pBSL45, respectively. These plasmids were digested with $S a c$ I and SalI, and the fragment was ligated to pHYG1 (a vector that contains the gfp gene) (Moriyama et al., 1999) that had been digested with $S a c$ I and SalI, yielding pHYS108G and pHYS45G, respectively, which contained the in-frame fusion of $g f p$ to $s l e B$.

Construction of cwlDgerE mutant To construct the cwlDgerE mutant, we used plasmid pBT374 (Sekiguchi et al., 1995). The plasmid was linearized with SalI and used to transform strain $\mathrm{AD} 17$ to chloramphenicol resistance $\left(\mathrm{Cm}^{\mathrm{r}}\right)$ by a double-crossover event, producing strain HF17.

Other procedures The microscopic fluorescence observations for GFP visualization were performed as described previously (Moriyama et al., 1999). 


\section{RESULTS}

Subcellular localization of SleB $_{1-108}$-GFP during sporulation To investigate the subcellular localization of SleB during sporulation, we fused the coding sequence for GFP in the frame to the 3' terminus of the sleB gene. The gene for GFP was joined to the 3' end of the 305 -codon sle $B$ open reading frame, and the resultant

A

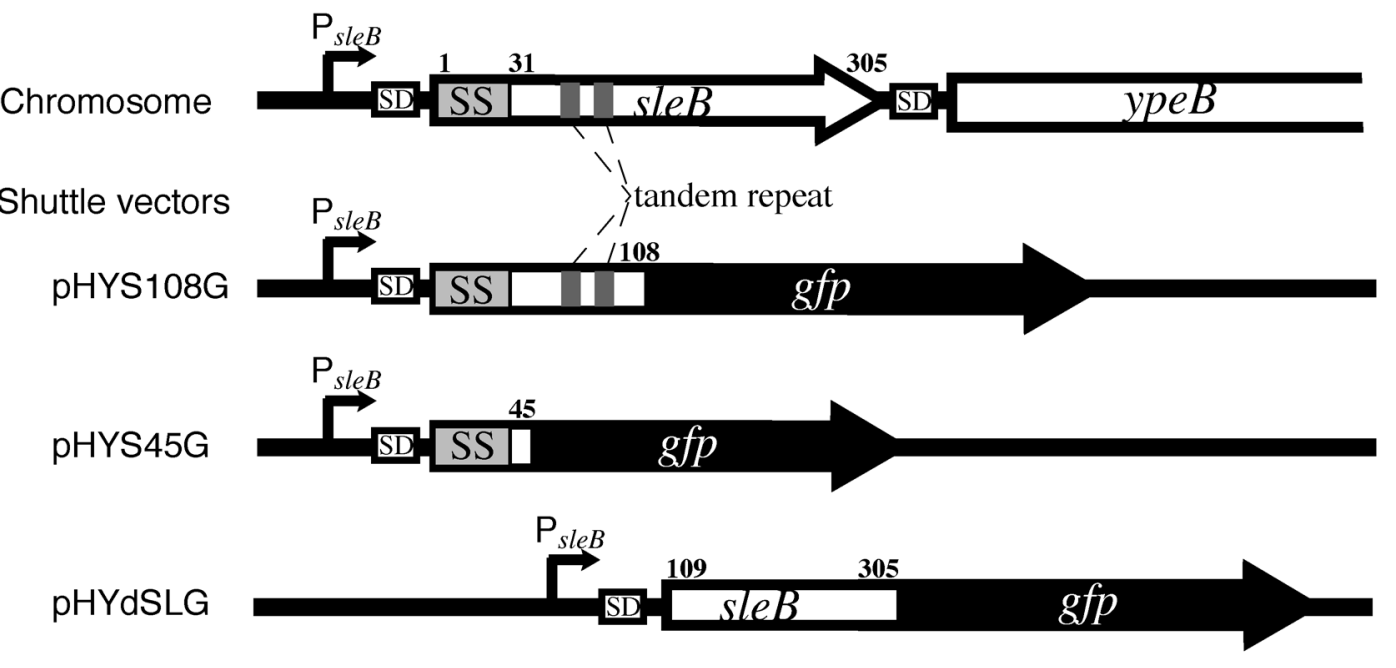

B

\begin{abstract}
signal peptide
\end{abstract}
B. anthracis 30-VEAFSNQVIQRGASGEDVIELQSRLKYNGFYTGKVDGVFGWGTYWALRNFQEKFGL-PVDGLAGAKTKQMLVKATKYDKSTANK - 112

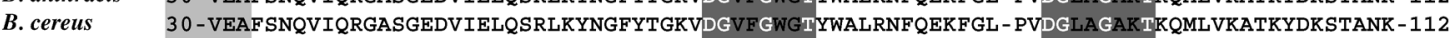

B. halodurans 27 -AHAF SDQVIQHGATGDDVVELQARLQYIGFYNKK IDGVFGWSTYWAVRNYQYEFGM-EVDGLVGPEMKAKLEKTTNFNRDFVDR - 109

B. megaterium 30 -AHAF SNQVIQHGAVGDDVIELQSRLQYLGYYNGK IDGVFGWSTYWALRNF QYEFGIKKIDGLAGWQTKIKLANATKYHENYVKN - 113

O. iheyensis 30 -TYAFSDQVIQQGAVGEDVIELQARLQYLGFYNGKIDGVFGWGTYWALRNFQYEFGM-EIDGLAGQTTKDKLVQASNYDKEYVTE-112

B. subtilis 27 -ISAFSNQVIQRGATGDDVVELQARLQYNGYYNGK IDGVYGWGTYWAVRNFQDQFGLKEVDGLVGAKTKQTLICKSKYYREYVME-110 pHYS45G

pHYS108G

Fig. 1. (A) Schematic representation of pHYS108G (expressing SleB $_{1-108}-\mathrm{GFP}$ ) and pHYS45G (expressing SleB-GFP 1-45 ). Shuttle vectors are derived from pHY300PLK (Takara) and constructed as described in Materials and Methods. SS (gray), signal sequence of SleB; gfp (black), gfp gene; SD (white), Shine-Dalgarno sequence. The tandem repeat sequences of SleB are shown in hatched boxes. (B) Protein sequence alignment of N-terminal regions of SleB homologues. B. subtilis (accession no. BAA11473), B. anthracis(accession no. NP_845098), B. cereus (accession no. BAA09800), B. halodurans (accession no. NP_242497), B. megaterium (accession no. BAE02885), Oceanobacillus iheyensis (accession no. P59105).
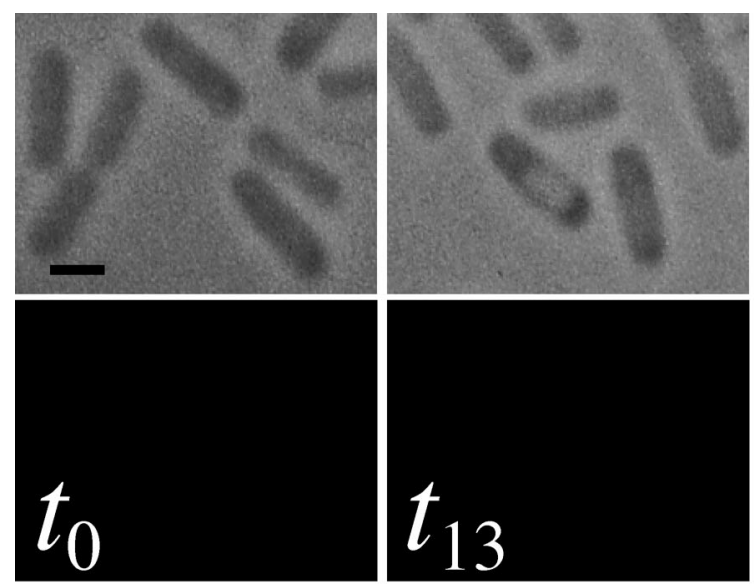
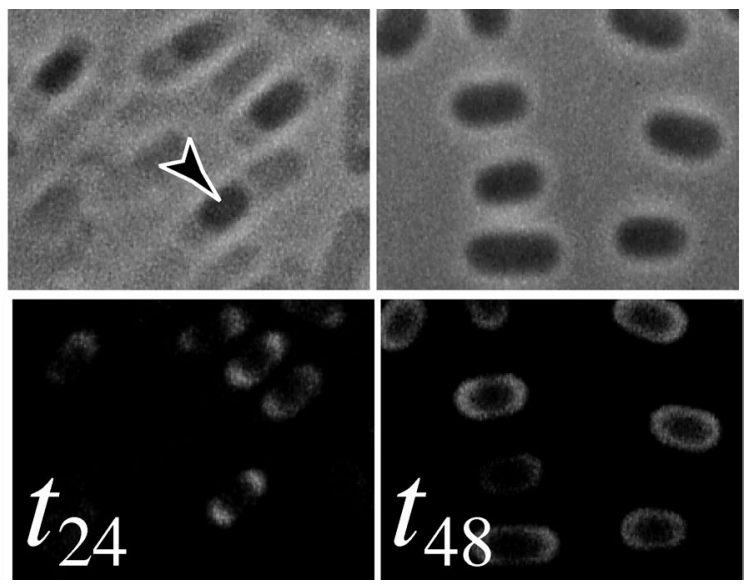

Fig. 2. Subcellular localization of $\mathrm{SleB}_{1-108}$-GFP in sporulating cells and dormant spores. Cells were sporulated by nutrient exhaustion as described in Materials and methods, and prepared for microscopy at approximately $0 \mathrm{~h}, 13 \mathrm{~h}, 24 \mathrm{~h}$ and $48 \mathrm{~h}$ after the onset of sporulation. Differential interference contrast micrographs (top panels) and fluorescence photographs (bot-

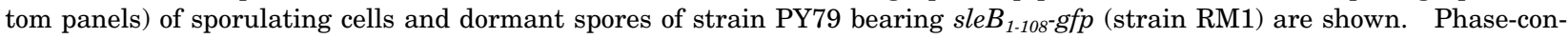
trast microscopic observation of the same samples was also carried out separately (data not shown). The arrowheads indicate the developing forespores in the top panels. Bar, $1 \mu \mathrm{m}$. 
plasmid was transformed into $B$. subtilis PY79 with selection for tetracycline resistance. However, when the transformant was sporulated in Difco sporulation medium containing tetracycline at either $30^{\circ} \mathrm{C}$ or $37^{\circ} \mathrm{C}$, no fluorescence was observed in the sporangia (data not shown), possibly because of impaired folding of the GFP moiety. Therefore, we constructed another fusion gene lacking the $\mathrm{C}$-terminal region of the $s l e B$ open reading
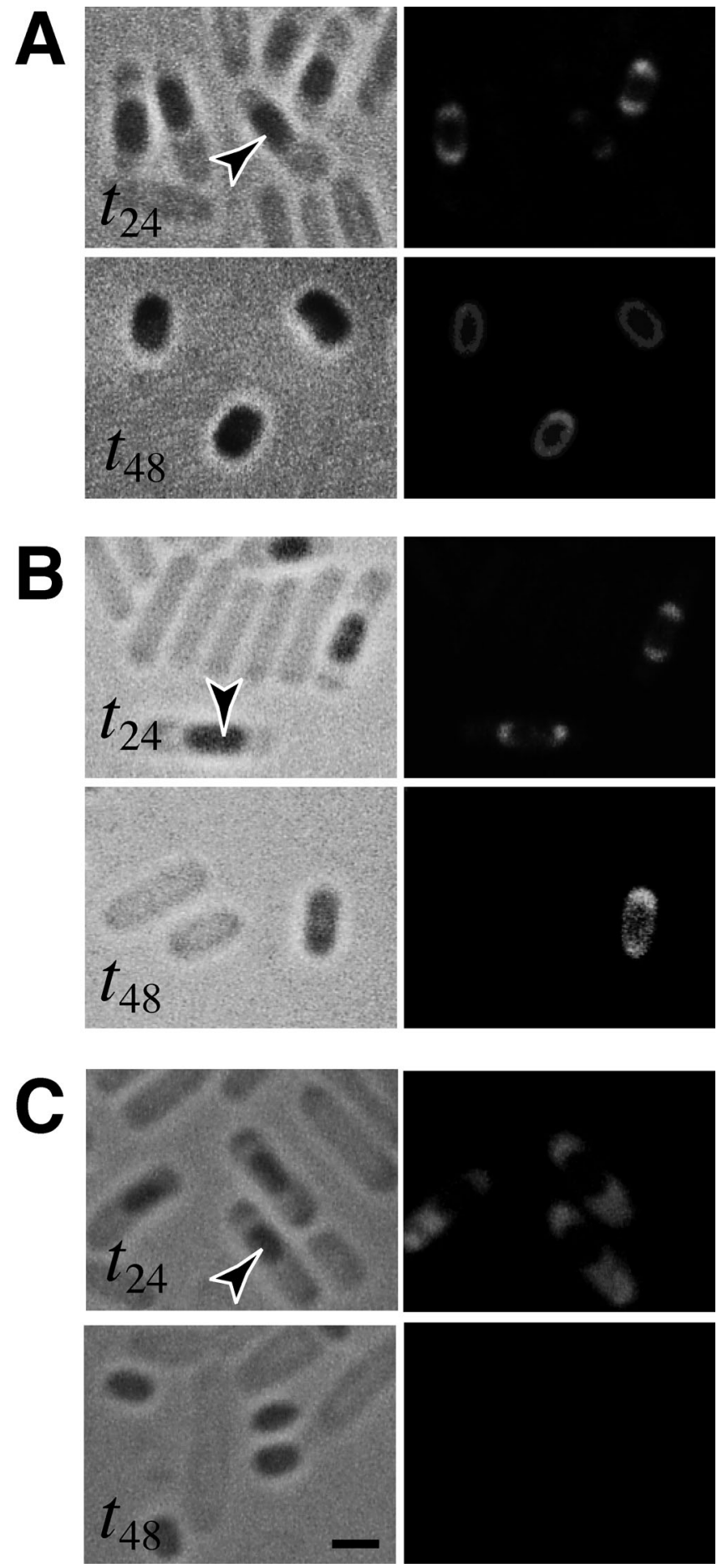

frame. The gene for GFP was joined to the 3' end of the 108-codon sleB open reading frame, which contains a secretion signal peptide and putative peptidoglycan binding domains (Fig. 1A). The resultant plasmid, pHYS108G, was transformed into B. subtilis PY79 with selection for tetracycline resistance, yielding strain RM1. Strain RM1 was sporulated at $30^{\circ} \mathrm{C}$ because no fluorescence was observed in sporangia sporulated at $37^{\circ} \mathrm{C}$, as reported previously (Moriyama et al., 1999).

As shown in Fig. 2, SleB $_{1-108}$-GFP exhibited a distinct pattern of the subcellular localization. In sporangia producing the $\mathrm{SleB}_{1-108}$-GFP fusion protein, the fluorescence appeared to surround the forespore as a ring at $t_{24}(24 \mathrm{~h}$ after the end of the exponential-growth phase). Phasecontrast microscopic observation suggested that sporulation stage of $t_{24}$ cells under this condition correspond to that of $\mathrm{B}$. subtilis $t_{7}$ cells which were sporulated at $37^{\circ} \mathrm{C}$ without antibiotic (Moriyama et al., 1999, Fukushima et al., 2002). At the point, more than $80 \%$ of sporangia possessed refractile forespores, indicating that at least some cortex had formed and that the forespores had partially dehydrated. SleB 1-108 GFP was also seen in the majority of free dormant spores at $t_{48}$. The fluorescence of $\mathrm{SleB}_{1}$ 108 -GFP was localized around the spore's periphery, as about $75 \%$ of the dormant spores were surrounded by $\mathrm{SleB}_{1-108}$-GFP. Previous works have shown that SleB is translocated by a secretion signal peptide from the forespore compartment and located in the spore cortex of the dormant spore (Moriyama et al., 1999, Chirakkal et al., 2002). Thus, the peripheral location of $\mathrm{SleB}_{1-108}-\mathrm{GFP}$ suggests the deposition of the fusion protein in the spore cortex synthesized between the inner and the outer forespore membranes, which is consistent with that of wildtype SleB.

Role of the tandem repeat motif of SleB for the enzyme localization As described above, it has been reported that various peptidoglycan hydrolases contain the characteristic tandem repeat sequences in their pri-

Fig. 3. Localization of $\mathrm{SleB}_{1-108}$-GFP and $\mathrm{SleB}_{1-45}$-GFP in sporulating cells and dormant spores of wild-type and gerE mutant strain. Sporulation was induced by nutrient exhaustion as described in Materials and methods, and samples were prepared for microscopy at approximately $24 \mathrm{~h}$ and $48 \mathrm{~h}$ after the onset of sporulation. Differential interference contrast micrographs (top panels) and fluorescence photographs (bottom panels) of sporulating cells and dormant spores of each strains bearing sleB-gfp are shown. (A) Sporulating cells and dormant spores of strain RM2 (sle $B_{1-45}-g f p$ ). (B and C) Sporangia of the gerE mutant, strains RM3 (AgerE sleB ${ }_{1-108}$-gfp) (B) and RM4 (AgerE $\left.s l e B_{1-45^{-}} g f p\right)$ (C), which synthesized the SleB-GFP fusion protein. Phase-contrast microscopic observation of the same samples was also carried out separately (data not shown). The arrowheads indicate the developing forespores in the top panels. Bar, $1 \mu \mathrm{m}$. 

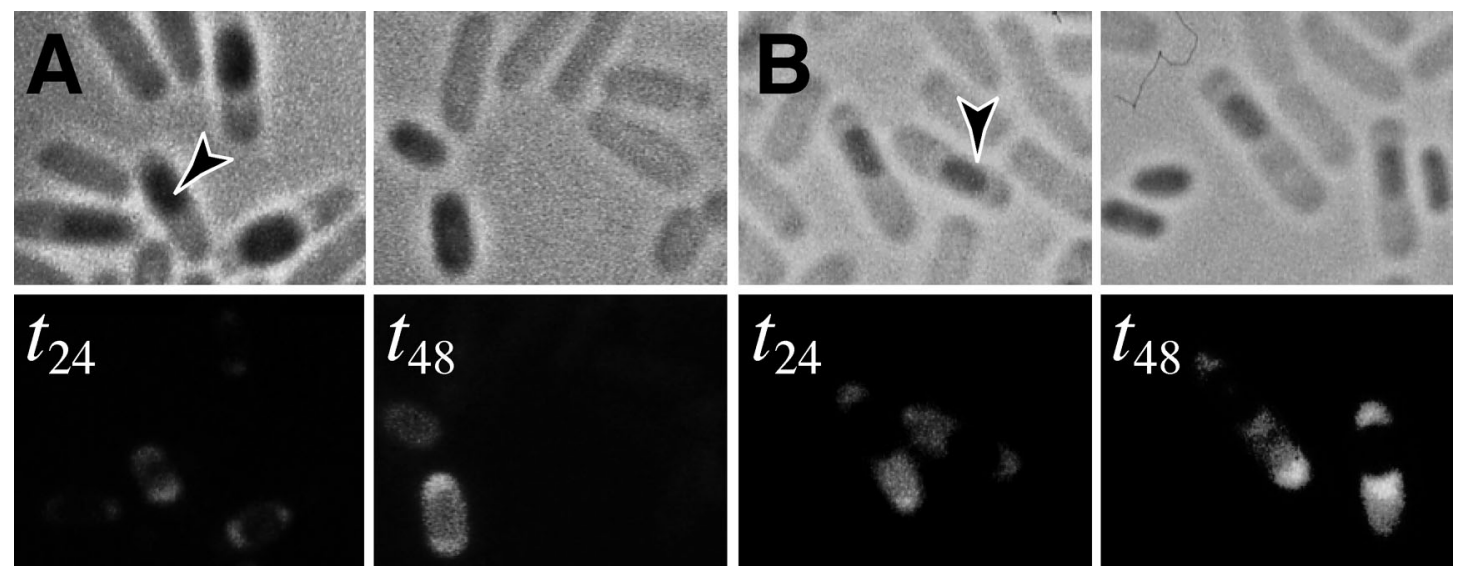

Fig. 4. Effect of muramic $\delta$-lactam defect on the localization pattern of SleB 1-108 $^{-G F P . ~(A ~ a n d ~ B) ~ F l u o r e s c e n c e ~ o f ~ s p o r u l a t i n g ~}$ cells and dormant spores of strains RM5 ( $\Delta c w l D$ sleB $\left.{ }_{1-108}-g f p\right)(\mathrm{A})$ and HF18 ( $\Delta c w l D g e r E$ sle $B_{1-108^{-}}$gfp) (B) that synthesized the fusion protein with the tandem repeat sequences. Phase-contrast microscopic observation of the same samples was also carried out separately (data not shown). Bar, $1 \mu \mathrm{m}$.

mary structure known as a putative peptidoglycan binding motif which might be involved in the recognition of substrate (Ghuysen et al., 1994). The motif is located in the N-terminal region of B. cereus and B. subtilis SleBs (Moriyama et al., 1996a, Moriyama et al., 1996b), as well as in the SleB homologs of other Bacillus species (Fig. 1B). To investigate whether the motif of SleB is involved in its localization during sporulation, we made a construct in which $g f p$ was fused to the $\mathrm{N}$-terminal 45 residues of SleB lacking the motif ( $\left.\mathrm{SleB}_{1-45}-\mathrm{GFP}\right)$, and the resultant plasmid, pHYS45G, was transformed into $B$. subtilis PY79, yielding strain RM2. In the sporangia of strain RM2, fluorescence encircled the forespore as in the case of $\mathrm{SleB}_{1-108}$-GFP (Fig. 3A). The results suggested that the signal peptides of both fusions are functional during sporulation. Next, we observed the localization patterns of both SleB1-108-GFP and SleB1-45-GFP in the gerE mutant. Since GerE regulates the activation of the late expression of spore coat genes, mutation leads to the production of lysozyme-sensitive spores with aberrant coat ultrastructure (Moir, 1981). In the sporangia of the gerE mutant, the fluorescence of $\mathrm{SleB}_{1-108}-\mathrm{GFP}$ surrounded the forespore as a ring at $t_{24}$ (Fig. 3B), and the localization pattern was similar to that in the wild-type strain. However, $\mathrm{SleB}_{1-45}$-GFP showed different localization patterns; the fusion protein diffused in the mother cell cytoplasm at $t_{24}$, and no fluorescence was detected in the dormant spores (Fig. 3C). These results suggested that the tandem repeat sequences of SleB are crucial in the enzyme localization, and that the coat layer, if it is formed normally, prevents the fusion without the motif from diffusing into a mother cell compartment, possibly as a physical barrier.

The muramic $\delta$-lactam structure of cortex is crucial for the proper localization of SleB In our pre- vious study, it was shown that SleB degrades only intact cortex but not the muramic $\delta$-lactam-deficient cortex (Makino et al., 1994), suggesting that muramic $\delta$-lactam is a major specific determinant of SleB as a germination lytic enzyme. Therefore, we assumed that muramic $\delta$ lactam might play an important role in the localization process of SleB as well as in the substrate specificity of the enzyme. To investigate this, we observed the localization pattern of $\mathrm{SleB}_{1-108}$-GFP in the $c w l D$ mutant (strain ADD1) which completely lacks muramic $\delta$-lactam in its spore cortex (Sekiguchi et al., 1995, Popham et al., 1996). As discussed above, however, there was a possibility that coat formation could prevent $\mathrm{SleB}_{1-108}-\mathrm{GFP}$ from dispersing into mother cell compartment, even if the fusion protein could not interact with the cortex peptidoglycan without $\delta$-lactam. In fact, $\mathrm{SleB}_{1-108}$-GFP encircled the forespore at $t_{24}$ and appeared as a ring around a $c w l D$ spore (Fig. 4A). So we constructed cwlDgerE mutant (strain HF17) which lacks both $\delta$-lactam structure in the spore cortex and many of spore coat proteins to evaluate a net importance of $\delta$-lactam in the localization of SleB during sporulation. Despite the presence of the repeat sequences, the fluorescence of the fusion was only observed in $15 \%$ of the sporangia at $t_{24}$ as a dot in the mother cell cytoplasm of the cwlDgerE mutant and no fluorescence was detected in the dormant spores (Fig. 4B). These results suggested that the synthesis of the muramic $\delta$-lactam during sporulation is crucial for the localization of SleB as well as the putative peptidoglycan binding motif of the enzyme.

\section{DISCUSSION}

In the present work, we demonstrated the subcellular localization of the spore cortex-lytic enzyme SleB during sporulation by using two fusions of $\mathrm{N}$-terminal region of 
SleB to GFP ( $\mathrm{SleB}_{1-108}-\mathrm{GFP}$ and $\left.\mathrm{SleB}_{1-45}-\mathrm{GFP}\right)$. The Nterminal region of $\mathrm{SleB}$ contains the repeat sequence motif (Moriyama et al., 1996b). The motif is composed of three -helices (Ghuysen et al., 1994) and classified in the putative peptidoglycan binding domain-1 [Pfam database]. This motif is conserved among many peptidoglycan hydrolases (e.g., SleC from $C$. perfringens (Miyata et al., 1995a), CwlA from B. subtilis (Kuroda and Sekiguchi, 1990), CwlL from B. licheniformis (Oda et al., 1993), DDcarboxypeptidase from Streptmyces albus (Joris et al., 1983), and autolytic lysozyme from C. acetobutylicum (Croux and Garcia, 1991)). Interestingly, when the importance of the motif in SleB localization was examined, we could discriminate the localization patterns of two fusion proteins in the gerE mutant (Fig. 3B and C) but not in the wild-type. The fluorescence of $\mathrm{SleB}_{1-45^{-}}$ GFP was diffused in the mother cell compartment in the gerE mutant (Fig. 3C), so we proposed that the coat layer might serve as a certain physical barrier for the fusion protein, and fluorescent ring formation by $\mathrm{SleB}_{1-45}-\mathrm{GFP}$ around the forespore of the wild-type (Fig. 3A) could be the artifact. Previously, Moir and Smith described that presumably the coat of the gerE spore is permeable to any soluble enzyme in the cortex that is free to diffuse (Moir, 1981, Moir and Smith, 1990), which is consistent with our results and supports above supposition. It should be noted, however, that $\mathrm{SleB}_{1-45}$-GFP in the gerE mutant after translocation across the inner forespore membrane was not trapped between the inner and outer forespore membrane where the spore peptidoglycan is synthesized. The integrity of the outer forespore membrane, which is ill-defined in electron micrographs, has been questioned (Craft-Lighty and Ellar, 1980) and there are some reports that the outer forespore membrane would not be effective permeability barrier in coat-defective spores (Driks, 1999, Cowan et al., 2004). Taken together with these reports, the diffusion of the fusion protein in the mother cell could be due to the dysfunction of both the outer forespore membrane and the coat layer as a barrier in coat-defective mutant.

The SleB-GFP fusion protein, even though it contained the repeat sequence motif, failed to localize normally in the coat- and muramic $\delta$-lactam-deficient strain (Fig. 4B), suggesting that the interaction between the peptidoglycan binding motif of SleB and the muramic $\delta$-lactam structure in the spore cortex are crucial to the proper localization of SleB. The peptidoglycan binding motif of SleB is conserved many peptidoglycan hydrolases as mentioned above, while each hydrolase varies in the substrate specificity. The germination-related enzymes, such as SleB and SleC, recognize and hydrolyze only peptidoglycan with muramic $\delta$-lactam moiety (Makino et al., 1994, Miyata et al., 1995a). The identification of the residues in the motif of the germination enzymes responsible for the recognition and/or binding to the muramic $\delta$-lactam structure requires further study.

From the results that SleB could not be detected immunochemically in the spore ingredients of ypeB mutant, Chirakkal et al. concluded that YpeB might be required for the localization and/or stabilization of SleB (Chirakkal et al., 2002). However, our results suggested that SleB after translocation across the inner forespore membrane could be localized in the spore cortex mediated by the interaction between the peptidoglycan binding motif of SleB and the muramic $\delta$-lactam in the spore cortex. In addition, SleB possesses a secretion Sec-type signal peptide (Moriyama et al., 1999, Tjalsma et al., 2000), and Imamura et al. have reported that a requisite cortex formation protein, SpoIVH, with a substitution of the SleB signal domain in place of its signal sequence was functional, suggesting that the translocation across the inner forespore membrane could be mediated only by the signal peptide (Imamura et al., 2004). Taken together, it might be possible that SleB could be localized properly in the spore cortex without any assistance from other spore proteins including YpeB. However, our results cannot exclude a possibility of the stabilization of SleB by YpeB. Further studies are needed to elucidate the roles of YpeB during sporulation. It will be also interesting in reference to the activation of SleB during spore germination that exists as a mature protein in the dormant spore.

We thank Adam Driks for providing B. subtilis strains PY79 and $\mathrm{AD} 17$, and Junichi Sekiguchi for providing B. subtilis strain ADD1 and plasmid pBT374.

\section{REERENCES}

Atrih, A., Zöllner, P., Allmaier, G., Williamson, M. P. and Foster, S. J. (1998) Peptidoglycan structural dynamics during germination of Bacillus subtilis 168 endospores. J. Bacteriol. 180, 4603-4612.

Boland, F. M., Atrih, A., Chirakkal, H., Foster, S. J., and Moir, A. (2000) Complete spore-corex hydrolysis during germination of Bacillus subtilis 168 requires SleB and YpeB. Microbiology 146, 57-64.

Chirakkal, H., O’Rourke, M., Atrih, A., Foster, S. J. and Moir, A. (2002) Analysis of spore cortex related proteins in Bacillus subtilis endospore germination. Microbiology 148, 23832392.

Cowan, A. E., Olivastro, E. M., Koppel, D. E., Loshon, C. A., Setlow, B. and Setlow, P. (2004) Lipids in the inner membrane of dormant spores of Bacillus species are largely immobile. Proc. Natl. Acad. Sci. USA 110, 7733-7738.

Craft-Lighty, A. and Ellar, D. J. (1980) The structure and function of the spore outer membrane in dormant and germinated spores of Bacillus megaterium. J. Appl. Bacteriol. 48, 135-145.

Croux, C. and Garcia, J. L. (1991) Sequence of the lyc gene encoding the autolytic lysozyme of Clostridium acetobutylcum ATCC824: comparison with other lytic enzymes. Gene. 104, 25-31.

Driks, A. (1999) Bacillus subtilis spore coat. Microbiol. Mol. Biol. Rev. 63, 1-20.

Fukushima, T., Yamamoto, H., Atrih, A., Foster, S. J., and 
Sekiguchi, J. (2002) A polysaccharide deacetylase gene $(p d a A)$ is required for germination and for production of muramic $\delta$-lactam residues in the spore cortex of Bacillus subtilis. J. Bacteriol. 184, 6007-6015.

Foster, S. J. and Johnstone, K. (1987) Purification and properties of a germination-specific cortex-lytic enzyme from spores of Bacillus megaterium KM. Biochem. J. 242, 573579.

Foster, S. J. and Johnstone, K. (1990) Pulling the trigger: the mechanism of bacterial spore germination. Mol. Microbiol. 4, 137-141.

Ghuysen, J. M., Lamotte-Brasseur, J., Joris, B. and Shockman, G. D. (1994) Binding site-shaped repeated sequences of bacterial wall peptidoglycan hydrolase. FEBS Lett. 342, 2328.

Imamura, D., Kobayashi, K., Sekiguchi, J., Ogasawara, N., Takeuchi, M. and Sato, T. (2004) spoIVH (ykvV), a requisite cortex formation gene, is expressed in both sporulating compartments of Bacillus subtilis. J. Bacteriol. 186, 54505459 .

Joris, B., van Beeumen, J., Casagrande, F., Gerday, C., Frere, J. M. and Ghuysen, J. M. (1983) The complete amino acid sequence of $\mathrm{Zn}^{2+}$-containing D-alanyl-D-alanine-cleaving carboxypeptidase of Streptomyces albus G. Eur. J. Biochem. 130, 53-69.

Kuroda, A. and Sekiguchi, J. (1990) Cloning, sequencing, and genetic mapping of Bacillus subtilis cell wall hydrolase gene. J. Gen. Microbiol. 136, 2209-2216.

Makino, S., Ito, N., Inoue, T., Miyata, S. and Moriyama, R. (1994) A spore-lytic enzyme released from Bacillus cereus spores during germination. Microbiology 140, 1403-1410.

Makino, S. and Moriyama, R. (2002) Hydrolysis of cortex peptidoglycan during bacterial spore germination. Med. Sci. Monit. 8, 119-127.

Miyata, S., Moriyama, R., Miyahara, N. and Makino, S. (1995a) A gene $(s l e C)$ encoding a spore cortex-lytic enzyme from Clostridium perfringens $\mathrm{S} 40$ spores; cloning, sequence analysis and molecular characterization. Microbiology 141, 2643-2650.

Miyata, S., Moriyama, R., Sugimoto, K. and Makino, S. (1995b) Purification and partial characterization of a spore cortexlytic enzyme of Clostridium perfringens S40 spores. Biosci.
Biotechnol. Biochem. 59, 514-515.

Moir, A. (1981) Germination properties of a spore coat-defective mutant of Bacillus subtilis. J. Bacteriol. 146, 1106-1116.

Moir, A. and Smith, D. A. (1990) The genetics of bacterial spore germination. Annu. Rev. Microbiol. 44, 531-553.

Moriyama, R., Hattori, A., Miyata, S., Kudoh, S. and Makino, S. (1996a) A gene (sleB) encoding a spore cortex-lytic enzyme from Bacillus subtilis and response of the enzyme to L-alanine-mediated germination. J. Bacteriol. 178, 6059-6063.

Moriyama, R., Kudoh, S., Miyata, S., Nonobe, S., Hattori, A. and Makino, S. (1996b) A germination-specific spore cortex-lytic enzyme from Bacillus cereus spores: cloning and sequencing of the gene and molecular characterization of the enzyme. J. Bacteriol. 178, 5330-5332.

Moriyama, R., Fukuoka, H., Miyata, S., Kudoh, S., Hattori, A., Kozuka, S., Yasuda, Y., Tochikubo, K. and Makino, S. (1999) Expression of a germination-specific amidase, SleB, of Bacilli in the forespore compartment of sporulating cells and its localization on the exterior side of the cortex in dormant spores. J. Bacteriol. 181, 2373-2378.

Oda, Y., Nakayama, R., Kuroda, A. and Sekiguchi, J. (1993) Molecular cloning, sequencing analysis, and characterization of a new cell wall hydrolase, CwlL, of Bacillus licheniformis. Mol. Gen. Genet. 241, 380-388.

Popham, D. L., Helin, J., Costello, C. E. and Setlow, P. (1996) Muramic lactam in peptidoglycan of Bacillus subtilis spores is required for spore outgrowth but not for spore dehydration or heat resistance. Proc. Natl. Acad. Sci. USA 93, 15405-15410.

Schaeffer, P., Millet, J. and Aubert, J. P. (1965) Catabolic repression of bacterial sporulation. Proc. Natl. Acad. Sci. USA 54, 704-711.

Sekiguchi, J., Akeo, K., Yamamoto, H., Khasanov, F. K., Alonso, J. C. and Kuroda, A. (1995) Nucleotide sequence and regulation of a new putative cell wall hydrolase gene, cwlD, which affects germination in Bacillus subtilis. J. Bacteriol. 177, 5582-5589.

Tjalsma, H., Bolhuis, A., Jongbloed, J. D. H., Bron, S. and van Dijl, J. M. (2000) Signal peptide-dependent protein transport in Bacillus subtilis: a genome-based survey of the secretome. Microbiol. Mol. Biol. Rev. 64, 515-547. 